\title{
RESISTENCIA Y SENSIBILIDAD BACTERIANA EN UROCULTIVOS EN UNA POBLACIÓN DE MUJERES DE ECUADOR
}

\author{
JOSÉ A. DURÁN CHÁVEZ1, ANDREA R. PÉREZ CASTILLO ${ }^{2}$, DENYS A. QUISPE ALCOCER ${ }^{2}$, \\ WENDY Y. GUAMÁN FLORES ${ }^{2}$, MARILIN. E JARAMILLO PUGA², DIANA E. ORMAZA BUITRÓN² \\ ${ }^{1}$ Doctor en Medicina General y Cirugía, Especialista en Ginecología y Obstetricia, Profesor titular de farmacología de \\ pregrado y posgrado Facultad de Ciencias Médicas, Universidad Central del Ecuador, Quito-Ecuador. \\ ${ }^{2}$ Médico General, Facultad de Ciencias Médicas, Universidad Central del Ecuador, Quito- \\ Ecuador. Hospital Básico PROVIDA. Unidades Materno- Fetal. Latacunga- Ecuador \\ Correspondencia: José Augusto Durán Chávez \\ jaduran_1975@yahoo.com
}

Recibido: 15 de marzo de 2018 Aceptado: 25 de julio de 2018

\section{Resumen}

Objetivo: Determinar la prevalencia de uropatógenos, sensibilidad y resistencia antimicrobiana en la infección del tracto urinario que acuden al Hospital Básico Privado "Provida" del 1 de enero de 2014 al 31 de diciembre de 2016. Material y métodos: Se analizaron los resultados de 116 urocultivos de orina en mujeres no gestantes de todas las edades de 2014 a 2016, que fueron atendidas en el Hospital Básico Privado "Provida" de la cuidad de Latacunga, en Ecuador. El análisis de los datos obtenidos se realizó mediante estadística descriptiva. Resultados: De las 116 muestras, se aislaron: Escherichia coli $(84,5 \%)$, Staphylococcus saprophyticus $(8,6 \%)$ y Proteus spp. $(6,9 \%)$. E. coli mostró sensibilidad a ceftriaxona en el $70 \%$, seguido de fosfomicina y gentamicina con el 62 y el $60 \%$, respectivamente. La sensibilidad hallada para quinolonas fue del $40 \%$ y la ampicilina sulbactam alcanzó el 37\%. Proteus spp. mostró sensibilidad del $75 \%$ para gentamicina y del $50 \%$ para quinolonas y cefuroxima. S. saprophyticus tuvo sensibilidad superior al $50 \%$ para gentamicina, ampicilina sulbactam, quinolonas y nitrofurantoína. Para E. coli la resistencia más alta registrada fue con ampicilina en el $86,5 \%$, seguido de las quinolonas con una resistencia superior al $50 \%$. La ampicilina asociada a inhibidor de betalactamasas, fosfomicina, cefalosporinas, nitrofurantoína y aminoglucósidos mostró resistencia inferior al 25\%. Conclusión: El agente patógeno más prevalente en infecciones del tracto urinario (ITU) es E. coli $(84,7 \%)$, porcentaje coincidente con lo reportado en la literatura nacional y mundial. Los antimicrobianos para este uropatógeno con mayor resistencia fueron ampicilina (86\%), cirprofloxacina (55\%) y norfloxacina (53\%). Se podría tener en cuenta en el momento de administrar una terapéutica empírica, dato que debería ser corroborado con información de susceptibilidades de acuerdo con el contexto.

Palabras clave: infección del tracto urinario; uropatógenos; prevalencia; sensibilidad; armacorresistencia microbiana 


\title{
BACTERIAL RESISTANCE AND SENSITIVITY IN URINE CULTURES IN A WOMEN POPULATION IN ECUADOR
}

\begin{abstract}
Summary
Objective: To determine the prevalence of uropathogens, sensitivity and antimicrobial resistance in urinary tract infections that go to the Private Basic Hospital "Provida" from January 1, 2014 to December 31, 2016. Material and methods: The results of 116 urine cultures in non-pregnant women of all ages from 2014 to 2016, which were treated at the Private Basic Hospital "Provida" of the city of Latacunga, in Ecuador, were analyzed. The data obtained was analyzed using descriptive statistics. Results: In the 116 samples, Escherichia coli (84.5\%), Staphylococcus saprophyticus $(8.6 \%)$ and Proteus spp. $(6.9 \%)$ were isolated. E. coli showed sensitivity to ceftriaxone in $70 \%$, followed by fosfomycin and gentamicin with 62 and $60 \%$, respectively. The sensitivity found for quinolones was $40 \%$ and for sulbactam ampicillin reached a $37 \%$. Proteus spp. showed sensitivity of $75 \%$ for gentamicin and $50 \%$ for quinolones and cefuroxime. S. saprophyticus had a sensitivity greater than $50 \%$ for gentamicin, sulbactam ampicillin, quinolones and nitrofurantoin. For E. coli the highest resistance recorded was found on ampicillin in $86.5 \%$, followed by quinolones with a resistance greater than $50 \%$. Ampicillin associated with inhibitor of beta-lactamase, fosfomycin, cephalosporins, nitrofurantoin and aminoglycosides showed a resistance below $25 \%$. Conclusion: The most prevalent pathogen in urinary tract infections (UTI) is E. coli (84.7\%), a percentage that matches what has been reported in national and world literature. The antimicrobials for this uropathogen with the highest resistance were ampicillin (86\%), cirprofloxacin (55\%) and norfloxacin (53\%). This should be taken into account when administering an empiric therapy, even though this data should be corroborated with the susceptibility information depending on the context.
\end{abstract}

Keywords: urinary tract infection; uropathogens; prevalence; sensitivity; microbial drug resistance

\section{RESISTÊNCIA E SENSIBILIDADE BACTERIANA EM UROCULTURAS NUMA POPULAÇÃO DE MULHERES DO EQUADOR}

\section{Resumo}

Objetivo: determinar a prevalência de uropatógenos, sensibilidade e resistência antimicrobiana na infecção do trato urinário que vão ao Hospital Básico Privado "Provida" de 1 de janeiro de 2014 a 31 de dezembro de 2016. Material e métodos: analisaram-se os resultados de 116 uroculturas de urina em mulheres não gestantes de todas as idades de 2014 a 2016, que foram atendidas no Hospital Básico Privado "Provida" da cidade de Latacunga, no Equador. A análise dos dados obtidos realizou-se mediante estatística descritiva. Resultados: das 116 amostras, isolaram-se: Escherichia coli (84,5\%), Staphylococcus saprophyticus $(8,6 \%)$ e Proteus spp. (6,9\%). E. coli mostrou sensibilidade a ceftriaxona em $70 \%$, seguido de fosfomicina e gentamicina com 62 e $60 \%$, respectivamente. A sensibilidade encontrada para quinolonas foi de $40 \%$ e a ampicilina sulbactam atingiu $37 \%$. Proteus spp. mostrou sensibilidade de $75 \%$ para gentamicina e de $50 \%$ para quinolonas e cefuroxima. S. saprophyticus teve sensibilidade superior a $50 \%$ para gentamicina, ampicilina sulbactam, quinolonas e nitrofurantoína. Para E. coli a resistência mais alta registrada foi com ampicilina em 86,5\%, seguido das quinolonas com uma resistência superior a 50\%. A ampicilina associada a inibidor de betalactamasas, fosfomicina, cefalosporinas, nitrofurantoína e aminoglucósidos mostrou resistência inferior a 25\%. Conclusão: o agente patogênico mais prevalente em infecções do trato urinário (ITU) é E. coli $(84,7 \%)$, porcentagem coincidente com o apresentado na literatura nacional e mundial. Os antimicrobianos para esse uropatógeno com maior resistência foram ampicilina (86\%), cirprofloxacina (55\%) e norfloxacina (53\%). Poderia ser tido em conta no momento de administrar uma terapêutica empírica, dado que deveria ser corroborado com informação de suscetibilidades de acordo com o contexto.

Palavras-chave: infecção do trato urinário; uropatógenos; prevalência; sensibilidade; resistência microbiana a medicamentos 


\section{Introducción}

La infección del tracto urinario (ITU) es un proceso inflamatorio sintomático por invasión, colonización y multiplicación de microorganismos en el tracto urinario inferior o superior (1-2). Es una de las enfermedades infecciosas más comunes a nivel comunitario. En los Estados Unidos, en 2007, 8,6 millones consultaron por ITU, de los cuales el $84 \%$ fueron mujeres (3), con una incidencia anual del $12 \%$, la mitad de todas las mujeres a los 32 años reportaron haber presentado al menos un episodio de ITU. No así en los hombres en quienes la incidencia es del $3 \%$, datos que se atribuyen a las diferencias en la anatomía: las mujeres tienen espacios periuretrales más húmedos, menor longitud de la uretra y mayor proximidad al ano (4)the incidence is high. Further, the potential impact of antibiotic treatment on the emergence of increasingly antibiotic-resistant UPEC (the resistant mechanisms that may be transmitted to other bowel inhabitants. Las manifestaciones clínicas relacionadas con ITU son frecuencia urinaria, disuria, urgencia miccional, nicturia, incontinencia urinaria, dolor lumbar, dolor abdominal; bajo estos síntomas suelen ser más graves en mujeres posmenopáusicas que en premenopáusicas (5).

En mujeres en edad fértil, los factores de riesgo para padecer de ITU incluyen vida sexual activa, uso de espermicidas, diafragmas vaginales e historia personal de infecciones previas. En posmenopáusicas, la incontinencia urinaria, la presencia de cistocele, el residuo posmiccional y el antecedente de cirugía ginecológica previa son factores predisponentes. Mientras que en mujeres mayores de 65 años se ha visto que las comorbilidades, la instrumentación urinaria y los problemas de vaciado (vejiga neurogénica) están relacionados con el desarrollo de ITU (6).

Los microorganismos que con mayor frecuencia son aislados por urocultivo son Escherichia coli $(46,4-74,2 \%)$, Klebsiella spp. (6,0-13,45\%), Enterobacter spp., Enterococcus spp. (5,3-9,54\%), Pseudomonas spp., Proteus spp. (4,7-11,9\%) y Staphylococcus saprophyticus (5-10\%), con diferencias según la población de estudio (7-8). Los estudios internacionales de vigilancia en poblaciones norteamericanas, europeas y sudamericanas estimaron que la prevalencia de E. coli resistente a nitrofurantoína fue del 8 y del $48 \%$ para ampicilina (9). En los Estados Unidos y Europa, la resistencia de E. coli a quinolonas es excepcional, y la resistencia al cotrimoxazol alcanza el $20 \%$ (10)ampicillin, ciprofloxacin, and nitrofurantoin among urine isolates of Escherichia coli from female outpatients, susceptibility testing data from The Surveillance Network (TSN. Las tasas de susceptibilidad en Brasil de los uropatógenos a ciprofloxacina y trimetoprim/sulfametoxazol es del 24,4 y del $50,6 \%$, respectivamente, mientras la susceptibilidad fue superior al $90 \%$ para la nitrofurantoína, los aminoglucósidos y las cefalosporinas de tercera generación (11). En Ecuador, el patógeno más frecuentemente aislado es E. coli. (12). Las propiedades genéticas de las bacterias, el uso irracional de antibióticos, la no adherencia al tratamiento, los viajes y el crecimiento poblacional contribuyen al aumento de la resistencia bacteriana a los antimicrobianos (7).

Las ITU altas tienden a desarrollar complicaciones con mayor frecuencia frente a las ITU bajas, en especial cuando no se instaura un tratamiento oportuno. Las principales complicaciones de las ITU son infecciones recurrentes especialmente en mujeres en edad reproductiva, cicatrización o daño renal permanente, abscesos intrarrenales, abscesos perinefríticos, pionefrosis y sindrome séptico, este último pone en riesgo la vida de las mujeres con ITU superior y es más común en mujeres de edad avanzada (13-14).

Las ITU constituyen un problema de salud pública por su elevada ocurrencia, morbilidad, alto costo de manejo, aumento de la tasa de recurrencia y la afectación de la calidad de vida de las pacientes tras cada nuevo episodio. La resistencia antimicrobiana tiene un efecto directo en el tratamiento individual del paciente, cuya terapéutica inicial es empírica, por tanto, es de vital importancia conocer la epidemiología y mantener el conocimiento actualizado sobre la susceptibilidad antimicrobiana de uropatógenos locales $(7-8,12)$.

La información existente en la actualidad en el Ecuador es escasa, y necesaria para generar un perfil clínico-epidemiológico propio, y que permita la implementación de medidas terapéuticas adecuadas a la realidad local, y a la vez contribuya a la disminución de la resistencia bacteriana.

\section{Material y métodos}

Se realizó un estudio descriptivo, observacional-transversal en el Hospital Privado Básico Provida de la cuidad de Latacunga, en Ecuador. Se analizaron los resultados de 116 urocultivos de orina de mujeres no gestantes en 
edades entre 7 y 84 años en el periodo comprendido entre el 1 de enero de 2014 al 31 de diciembre de 2016.

Los resultados analizados fueron de urocultivos positivos de más de 100000 unidades formadoras de colonia (UFC).

El análisis de datos se realizó con el programa SPSS v23.00, se utilizó estadística descriptiva para los resultados de urocultivos, resistencia y susceptibilidad bacteriana.

\section{Resultados}

De un total de 116 muestras consideradas como positivas, se obtuvieron los resultados que se describen a continuación.

Se aislaron: E. coli, Proteus spp. y S. saprophyticus, el patógeno más frecuentemente aislado fue E. coli en el 84,5\% de las muestras (tabla 1).

Tabla 1. Frecuencia por bacteria

\begin{tabular}{|c|c|c|}
\hline Bacteria & $\begin{array}{c}\text { Frecuencia } \\
\text { (n) }\end{array}$ & $\begin{array}{c}\text { Porcentaje } \\
(\mathbf{\%})\end{array}$ \\
\hline Escherichia coli & 98 & 84,5 \\
\hline Staphylococcus saprophyticus & 10 & 8,6 \\
\hline Proteus spp. & 8 & 6,9 \\
\hline Total & $\mathbf{1 1 6}$ & $\mathbf{1 0 0 , 0}$ \\
\hline
\end{tabular}

Fuente: Elaboración propia.

El $68,1 \%$ de los resultados de urocultivos pertenecían a mujeres en edades de 19 a 41 años, y tan solo el 3,4\% a menores de 18 años (tabla 2).

Tabla 2. Frecuencia según la edad

\begin{tabular}{|c|c|c|}
\hline Edad & $\begin{array}{c}\text { Frecuencia } \\
\text { (n) }\end{array}$ & $\begin{array}{c}\text { Porcentaje } \\
\text { (\%) }\end{array}$ \\
\hline$\leq 18$ & 4 & 3,4 \\
\hline $19-41$ & 79 & 68,1 \\
\hline $42-64$ & 25 & 21,6 \\
\hline$\geq 65$ & 8 & 6,9 \\
\hline Total & $\mathbf{1 1 6}$ & $\mathbf{1 0 0}$ \\
\hline
\end{tabular}

Fuente: Elaboración propia.
En todos los grupos etarios, la bacteria predominante fue E. coli con el $84 \%$. S. saprophyticus se encontró en un $90 \%$ en mujeres de 19 a 41 años (tabla 3).

Tabla 3. Frecuencia bacterias reportadas según edad

\begin{tabular}{|c|c|c|c|c|c|c|c|c|}
\hline & \multicolumn{4}{|c|}{ Gramnegativos } & \multirow{2}{*}{\multicolumn{2}{|c|}{\begin{tabular}{|c|} 
Grampositivos \\
$\begin{array}{c}\text { Staphylococcus } \\
\text { saprophyticus }\end{array}$ \\
\end{tabular}}} & & \\
\hline & \multicolumn{2}{|c|}{ Escherichia coli } & \multicolumn{2}{|c|}{ Proteus spp. } & & & \multicolumn{2}{|c|}{ Total } \\
\hline & 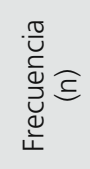 & 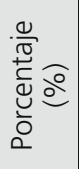 & 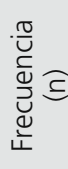 & 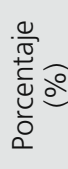 & 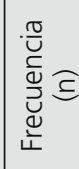 & 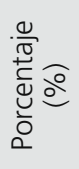 & 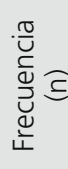 & 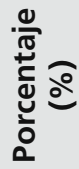 \\
\hline$\leq 18$ & 3 & 3 & 1 & 12,5 & 0 & 0 & 4 & 3,4 \\
\hline $19-41$ & 66 & 67 & 4 & 50 & 9 & 90 & 79 & 68,1 \\
\hline $42-64$ & 21 & 21 & 3 & 37,5 & 1 & 10 & 25 & 21,5 \\
\hline$\geq 65$ & 8 & 8 & 0 & 0 & 0 & 0 & 8 & 6,8 \\
\hline Total & 98 & 100 & 8 & 100 & 10 & 100 & 116 & 100 \\
\hline
\end{tabular}

Fuente: Elaboración propia.

E. coli mostró sensibilidad a ceftriaxona en el $70 \%$, seguido de fosfomicina y gentamicina con el 62 y el $60 \%$, respectivamente. La sensibilidad hallada para quinolonas fue del $40 \%$, mientras que para ampicilina la sensibilidad fue del $1 \%$, pero la combinación ampicilina/sulbactam alcanzó el 37\% (tabla 4).

Tabla 4. Frecuencia según sensibilidad

\begin{tabular}{|c|c|c|c|c|c|c|}
\hline \multirow[b]{3}{*}{ Antibiótico } & \multicolumn{4}{|c|}{ Gramnegativos } & \multirow{2}{*}{\multicolumn{2}{|c|}{$\begin{array}{c}\text { Grampositivos } \\
\text { Staphylococcus } \\
\text { saprophyticus }\end{array}$}} \\
\hline & \multicolumn{2}{|c|}{$\begin{array}{c}\text { Escherichia } \\
\text { coli }\end{array}$} & \multicolumn{2}{|c|}{ Proteus spp. } & & \\
\hline & 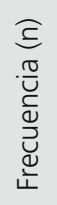 & 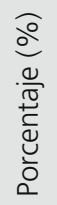 & 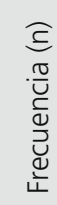 & $\begin{array}{l}\frac{\widehat{0}}{0} \\
\frac{0}{0} \\
\frac{0}{0} \\
\frac{0}{0} \\
0\end{array}$ & 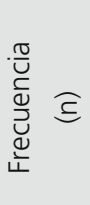 & 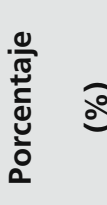 \\
\hline Ceftriaxona & 68 & 70 & 3 & 37 & - & - \\
\hline Fosfomicina & 61 & 62 & 1 & 12 & - & - \\
\hline Gentamicina & 59 & 60 & 6 & 75 & 7 & 70 \\
\hline Cefuroxima & 54 & 55 & 4 & 50 & - & - \\
\hline Nitrofurantoína & 52 & 53 & - & - & 8 & 80 \\
\hline Ciprofloxacina & 39 & 40 & 4 & 50 & 5 & 50 \\
\hline Norfloxacina & 39 & 40 & 4 & 50 & 4 & 40 \\
\hline
\end{tabular}




\begin{tabular}{|c|c|c|c|c|c|c|}
\hline \multirow[b]{3}{*}{ Antibiótico } & \multicolumn{4}{|c|}{ Gramnegativos } & \multirow{2}{*}{\multicolumn{2}{|c|}{$\begin{array}{c}\text { Grampositivos } \\
\text { Staphylococcus } \\
\text { saprophyticus }\end{array}$}} \\
\hline & \multicolumn{2}{|c|}{$\begin{array}{l}\text { Escherichia } \\
\text { coli }\end{array}$} & \multicolumn{2}{|c|}{ Proteus spp. } & & \\
\hline & 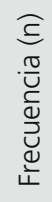 & 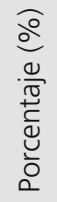 & 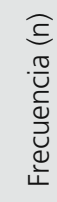 & $\begin{array}{l}\frac{0}{0} \\
\frac{d}{\frac{0}{0}} \\
\frac{ \pm}{0} \\
\frac{0}{0} \\
0\end{array}$ & 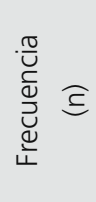 & 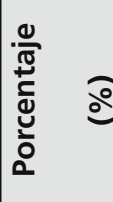 \\
\hline $\begin{array}{l}\text { Ampicilina/ } \\
\text { sulbactam }\end{array}$ & 36 & 37 & 1 & 12 & 9 & 90 \\
\hline Ampicilina & 1 & 1 & 一 & - & 3 & 30 \\
\hline $\begin{array}{l}\text { Trimetoprima/ } \\
\text { sulfametoxazol }\end{array}$ & 1 & 1 & 一 & - & 一 & - \\
\hline Total & 97 & & 8 & & 10 & \\
\hline
\end{tabular}

Fuente: Elaboración propia.

Proteus spp. mostró sensibilidad del $75 \%$ para gentamicina y del $50 \%$ para quinolonas y cefuroxima. S. saprophyticus tuvo sensibilidad superior al $50 \%$ para gentamicina, ampicilina/sulbactam, quinolonas y nitrofurantoína (tabla 4).

Para E. coli la resistencia más alta registrada fue con ampicilina en el $86 \%$ seguido de quinolonas con un porcentaje de resistencia superior al 50\%. Ampicilina/ sulbactam, fosfomicina, cefalosporinas de segunda $y$ tercera generación, nitrofurantoína y aminoglucósidos mostraron resistencia inferior al $25 \%$. La resistencia de ampicilina en $S$. saprophyticus es del $60 \%$. Proteus spp. resistente a fosfomicina en el $62 \%$ (tabla 5).

Tabla 5. Frecuencia según resistencia

\begin{tabular}{|c|c|c|c|c|c|c|}
\hline \multirow[b]{3}{*}{ Antibiótico } & \multicolumn{4}{|c|}{ Gramnegativos } & \multirow{2}{*}{\multicolumn{2}{|c|}{$\begin{array}{c}\text { Grampositivos } \\
\text { Staphylococcus } \\
\text { saprophyticus }\end{array}$}} \\
\hline & \multicolumn{2}{|c|}{$\begin{array}{l}\text { Escherichia } \\
\text { coli }\end{array}$} & \multicolumn{2}{|c|}{$\begin{array}{c}\text { Proteus } \\
\text { spp. }\end{array}$} & & \\
\hline & 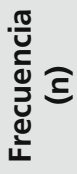 & 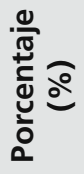 & 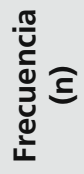 & 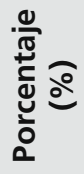 & 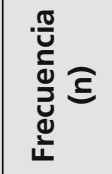 & 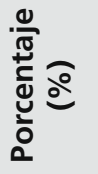 \\
\hline Ampicilina & 77 & 86 & - & - & 6 & 60 \\
\hline Ciprofloxacina & 49 & 55 & 2 & 25 & 3 & 30 \\
\hline Norfloxacina & 48 & 53 & 3 & 37 & 3 & 30 \\
\hline Fosfomicina & 24 & 27 & 5 & 62 & - & - \\
\hline $\begin{array}{l}\text { Ampicilina/ } \\
\text { sulbactam }\end{array}$ & 20 & 22 & 4 & 50 & 0 & 0 \\
\hline
\end{tabular}

\begin{tabular}{|c|c|c|c|c|c|c|}
\hline & \multicolumn{4}{|c|}{ Gramnegativos } & \multirow{2}{*}{\multicolumn{2}{|c|}{$\begin{array}{l}\text { Grampositivos } \\
\text { Staphylococcus } \\
\text { saprophyticus }\end{array}$}} \\
\hline & \multicolumn{2}{|c|}{$\begin{array}{c}\text { Escherichia } \\
\text { coli }\end{array}$} & \multicolumn{2}{|c|}{$\begin{array}{c}\text { Proteus } \\
\text { spp. }\end{array}$} & & \\
\hline Antibiótico & 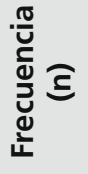 & 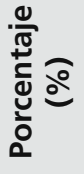 & 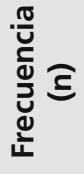 & 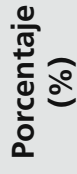 & 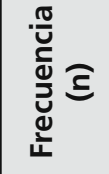 & 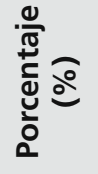 \\
\hline Ceftriaxona & 20 & 22 & 4 & 50 & - & - \\
\hline Cefuroxima & 18 & 20 & 4 & 50 & - & - \\
\hline Nitrofurantoína & 11 & 12 & - & - & 1 & 10 \\
\hline Gentamicina & 11 & 12 & 2 & 25 & 1 & 10 \\
\hline $\begin{array}{l}\text { Trimetoprima/ } \\
\text { sulfametoxazol }\end{array}$ & 1 & 1 & - & - & - & - \\
\hline Total & 89 & 83 & 8 & 7,5 & 10 & 9,3 \\
\hline
\end{tabular}

Fuente: Elaboración propia.

\section{Discusión}

En este estudio, el uropatógeno aislado con mayor frecuencia fue E. coli $(84,5 \%)$, porcentaje similar al obtenido en estudios internacionales del 73-94\% (15-18). S. Saprophyticus y Proteus spp. presentaron una frecuencia del 8,6 y del 6,9\%, respectivamente, lo que concuerda con el estudio SENTRY realizado en Argentina, Chile, Brazil, México y Venezuela (19). Según Marchisio et al. y Cunha et al., la prevalencia de Klebsiella spp. es del 9-14\%, dato que no coincide con nuestro hallazgo, por lo que es probable que existan factores que limiten la identificación o el crecimiento de este uropatógeno, que se deberían determinar $(11,20)$.

En el estudio brasileño de Rocha et al., que incluyó mujeres con edades entre 13 y 81 años aproximadamente, determinó que en el grupo de edad entre 21-30 años la ITU es más prevalente y que luego disminuye progresivamente con el aumento de la edad a un mínimo de 3,3 en la edad >81 años (21), datos comparables con los hallazgos de este estudio, en los que el rango de mayor probabilidad para aislar un uropatógeno fue entre 19 y 41 años, no así en adultas mayores en las que la frecuencia es del 6,9\%. Guamán et al. condujeron un estudio sobre resistencia bacteriana para E. coli en población ecuatoriana, el cual determinó que el grupo etario más susceptible de presentar una ITU estaba entre los 11 a 20 años $(28,3 \%)(22)$. 
La sensibilidad más alta de E. coli reportada en este estudio fue para ceftriaxona (70\%), seguido de fosfomicina (62\%), gentamicina (60\%), cefuroxima (55\%) y nitrofurantoína (53\%). Datos que concuerdan con otro estudio local en el que las cefalosporinas mostraron una tasa mayor del $80 \%$ de susceptibilidad (22). Según Rocha, la susceptibilidad de E. coli para nitrofurantoína y cefalosporinas de amplio espectro es mayor en comparación con la susceptibilidad para otros patógenos que causan ITU (21). En el estudio de Salles, llevado a cabo en América Latina, las tasas de sensibilidad reportadas fueron similares a las encontradas en este trabajo (18). En nuestra investigación, la sensibilidad a quinolonas es del $40 \%$, mientras que en el estudio brasileño de Cunha et al. alcanza el 75\% (11).

Con respecto a los patrones de resistencia a los antimicrobianos de uso común en el tratamiento de ITU, se encontró que para el manejo de las infecciones causadas por E. coli la ampicilina no es una buena opción terapéutica, la tasa de resistencia es del $86,5 \%$, al asociarla con un betalactámico disminuye al $22,5 \%$, mientras que las quinolonas presentan valores superiores al $50 \%$. Según OrregoMarin, la resistencia a fluoroquinolonas es alrededor del $42 \%$, valor aproximado al obtenido en este trabajo (13). En el estudio ecuatoriano de Guamán et al., la resistencia a ampicilina es del $52,5 \%$ y de quinolonas superior al $25 \%$, valores inferiores a lo hallado (22). Para trimetoprim/, la resistencia reportada es del $1,1 \%$ dato que no concuerda con otros estudios en los que la resistencia contra este fármaco supera el $40 \%(13,15-19,21)$, debido a que en los resultados analizados únicamente utilizaron un urocultivo.

\section{Conclusiones}

El agente patógeno más prevalente en ITU es E. coli $(84,5 \%)$, valor coincidente con lo reportado en la literatura nacional y mundial. Los datos de susceptibilidad antimicrobiana en nuestro estudio en su mayoría son concordantes con los resultados obtenidos en otros trabajos similares. Para E. coli los antimicrobianos con mayor resistencia fueron ampicilina (86\%), cirprofloxacina (55\%) y norfloxacina (53\%), lo que se podría tener en cuenta en el momento de administrar una terapéutica empírica, dato que debería ser corroborado con información de susceptibilidades de acuerdo con el contexto. En este estudio, existen limitaciones en la identificación de la especie de Proteus, por lo que nuestros datos respecto de otros difieren.

\section{Contribución de los autores}

El protocolo de investigación y el diseño de esta, la recolección de datos, el análisis estadístico, la valoración e interpretación de los datos, el análisis crítico, la discusión, redacción y la aprobación del artículo final fueron realizados por todos los autores quienes contribuyeron de igual forma a lo largo del proceso. El autor correspondiente representa al colectivo de autores.

\section{Disponibilidad de datos y materiales}

Los datos que sustentan este artículo están disponibles bajo requisición al autor correspondiente.

\section{Consentimiento para publicación}

La identidad de los individuos participantes en el estudio es anónima y confidencial, por lo que no se obtuvo un consentimiento específico para su publicación. Cabe mencionar que según la Resolución 8430 de 1993 es una investigación sin riesgo.

\section{Agradecimientos}

Los autores agradecen a todas las personas que colaboraron en el proceso de la investigación. Al personal médico y administrativo del Hospital Básico Provida.

\section{Referencias}

1. Hernández-Burruezo JJ, Mohamed-Balghata MO, Martínez LA, et al. Infecciones del aparato urinario. Medicina Clínica. 2007;129(18):707-715. https://doi. org $/ 10.1157 / 13112512$

2. Nzalie RNT, Gonsu HK, Koulla-Shiro S. Bacterial etiology and antibiotic resistance profile of communityacquired urinary tract infections in a cameroonian city. Int J Microbiol. 2016;2016:1-6. http://dx.doi. org $/ 10.1155 / 2016 / 3240268$

3. Schappert SM, Rechtsteiner EA. Ambulatory medical care utilization estimates for 2007. Vital Health Stat. [internet] 2011 abr [citado 2019 mrz 15]; 13(169):1-38. Disponible en: https://www.cdc.gov/nchs/data/series/sr_13/ sr13_169.pdf

4. Foxman B, Brown P. Epidemiology of urinary tract infections: Transmission and risk factors, incidence, and costs. Infect Dis Clin North Am. 2003;17(2):227-41. DOI: 10.1016/S0891-5520(03)00005-9

5. Arinzon Z, Shabat S, Peisakh A, et al. Clinical presentation of urinary tract infection (UTI) differs with aging in women. Arch Gerontol Geriatr. 2012;55(1):145-7. https://doi. org/10.1016/j.archger.2011.07.012 
6. Alós JI. Epidemiología y etiología de la infección urinaria comunitaria: sensibilidad antimicrobiana a determinados patógenos y significado clínico de la resistencia. Medes. 2005;23(4):3-8.

7. Nozarian Z, Abdollahi A. Microbial etiology and antimicrobial susceptibility of bactria implicated in urinary tract infection in Tehran, Iran. Iran J Pathol. 2015 [citado 2019 mrz 15]; 10(1):54-60. Disponible en: https://www.ncbi.nlm. nih.gov/pmc/articles/PMC4539787/

8. Linhares I, Raposo T, Rodrigues A, Almeida A. Frequency and antimicrobial resistance patterns of bacteria implicated in community urinary tract infections: a ten-year surveillance study (2000-2009). BMC Infect Dis. 2013;13(1):19. https:// doi.org/10.1186/1471-2334-13-19

9. Wong CKM, Kung K, Au-Doung PLW, et al. Antibiotic resistance rates and physician antibiotic prescription patterns of uncomplicated urinary tract infections in southern Chinese primary care. PloS one. 2017; 12(5):e0177266. https://doi.org/10.1371/journal.pone.0177266

10. Karlowsky JA, Kelly LJ, Thornsberry C, et al. Trends in antimicrobial resistance among urinary tract infection isolates of Escherichia coli from female outpatients in the United States. Antimicrob Agents Chemother. 2002;46(8):25405. DOI: 10.1128/AAC.46.8.2540-2545.2002

11. Cunha MA, Assunção GLM, Medeiros IM, et al. Antibiotic resistance patterns of urinary tract infections in a northeastern Brazilian capital. Rev Inst Med Trop Sao Paulo. 2016;58:2-5. http://dx.doi.org/10.1590/S16789946201658002

12. Zurita Salina J. Epidemiología de la resistencia bacteriana en el Ecuador. En: Uso racional de antibióticos; 2004. p. 26-41.

13. Orrego-Marin CP, Henao-Mejía CP, Cardona-Arias JA. Prevalencia de infección urinaria, uropatógenos y perfil de susceptibilidad antimicrobiana. Acta Medica Colomb. [internet] 2014 oct-dic [citado 2019 mrz 15];39(4):352-8. Disponible en: https://www.redalyc. org/html/1631/163132885008/

14. Gonzáles Monte E. Infecciones de tracto urinario. Nefrol al Día. [internet] 2012 oct [citado 2019 mrz 15]; 26(1):323. http://revistanefrologia.com/es-monografias-nefrologiadia-articulo-infecciones-tracto-urinario-4
15. Sivick KE, Mobley HLT. Waging war against uropathogenic Escherichia coli: Winning back the urinary tract. Infect Immun. 2010;78(2):568-85. DOI: 10.1128/IAI.01000-09

16. Martins F, Vitorino J, Abreu A. Avaliação do perfil de susceptibilidade aos antimicrobianos de microrganismos isolados em urinas: Na Região do Vale do Sousa e Tâmega. Acta Med Port. [internet] 2010 nov-dic [citado 2019 mrz 15]; 23(4):641-6. https://www.actamedicaportuguesa. com/revista/index.php/amp/article/download/5352/41 $54+\& c d=1 \& h l=e s \& c t=c \ln \& g l=e c$

17. Gallardo Luna MG, Magaña Aquino M, Andrade Rodríguez HJ, Jiménez De La Torre MJ, Sánchez Álvarez K, Fragoso Morales LE. Resistencia a fármacos empleados en infección de vías urinarias en pacientes de primer contacto en una Unidad de Medicina Familiar del IMSS. Enfermedades Infecc y Microbiol. [internet] 2008 [citado $2019 \mathrm{mrz}$ 15];28(1):13-8. http://www.medigraphic.com/cgi-bin/ new/resumen.cgi?IDARTICULO $=26633$

18. Salles MJC, Zurita J, Mejía C, Villegas MV. Resistant Gramnegative infections in the outpatient setting in Latin America. Epidemiol Infect. 2013;141(12):2459-72. https://doi. org/10.1017/S095026881300191X

19. Andrade SS, Sader HS, Jones RN, Pereira AS, Pignatari ACC, Gales AC. Increased resistance to first-line agents among bacterial pathogens isolated from urinary tract infections in Latin America: Time for local guidelines? Mem Inst Oswaldo Cruz. 2006;101(7):741-8. http://dx.doi. org/10.1590/S0074-02762006000700006

20. Marchisio M, Porto A, Joris R, Rico M, Baroni MR, Di Conza J. Susceptibility to $\beta$-lactams and quinolones of enterobacteriaceae isolated from urinary tract infections in outpatients. Brazilian J Microbiol. 2015;46(4):1155-9. http:// dx.doi.org/10.1590/S1517-838246420140880

21. Rocha JL, Tuon FF, Johnson JR. Sex, drugs, bugs, and age: rational selection of empirical therapy for outpatient urinary tract infection in an era of extensive antimicrobial resistance. Braz J Infect Dis. 2012;16(2):115-121.

22. Guamán WM, Tamayo VR, Villacís JE, et al. Resistencia bacteriana de Escherichia coli uropatogénica en población nativa amerindia Kichwa de Ecuador. Rev Fac Cien Med. 2017;42(1):37-46. 